Lunar Mineralogy. By Judith W. Frondel. Pp. $\mathrm{x}+323$. (Wiley-Interscience: New York and London, October 1975.) £11.20.

THE ending of the Apollo and Luna missions marks a significant turning point in the development of lunar science. Over the past five years efforts have concentrated on frantic reconnaissance and sample analysis in preparation for future missions, but now the pace has changed and problemorientated studies and a more thoughtful evaluation of published data seem to be the most obvious of immediate future developments. In this situation the appearance of a book of this type is particularly valuable. About 850 pounds of rock were recovered in the Apollo programme, but it is important to remember that of this only about $10 \%$ has been extensively examined; approximately 20,000 individually identified samples remain available for future study.

This book has grown from two editions of a Glossary of Lunar Materials which the author prepared for distribution by NASA to principal investigators of the Apollo programme. It is arranged in the manner of Dana's System of Mineralogy and summarises all lunar mineralogical data published up to and including the Fifth Lunar Science Conference in March 1974. The author's task has been aided by the Earth's satellite, for rich repositories of rare and spectacular minerals such as pegmatites, true granites and hydrous vein deposits are conspicuously absent in the Moon; the rocks remain in a highly reduced state and there is a marked depletion in elements more volatile than iron. It is not surprising therefore to learn that only 60 valid and 14 tentatively identified mineral phases have so far been reported compared with about 2,200 on Earth; and there are no lunar diamonds.

Individual minerals are described in eleven systematic chapters; the treatment here is concise but adequate, and each section gives a good account of how particular minerals occur in lunar rocks. There are useful tables with selections of mineral analyses, photomicrographs wherever possible, and abbreviated references. The wisdom of including here abbreviated optical and X-ray data on minerals such as olivines is questionable; the photomicrographs and specimen photographs are of a high quality, and the eye is captivated by the beautiful scanning electron microscope photographs of iron crystals deposited in vugs. The systematic chapters are pre ceded by an excellent 16-page introduc- tion which summarises the history and petrology of the lunar surface; this section can be thoroughly recommended to amateurs. With these in mind, however, it is disturbing to read on page 7 that "Total and partial melting, a relatively insignificant feature on Earth, is important in the petrogenesis of the lunar highlands".

The author and publishers are to be congratulated on the high standard of production. The book will be a standard reference for many years to come, and will be particularly valuable for those who have not worked on lunar minerals. The main text is supported by a bibliography, simple index and appendices which list the samples mentioned in the text and explain the sample-numbering system.

I. D. Muir

\section{Lunar science}

Lunar Science: A Post-Apollo ViewScientific Results and Insights from the Lunar Samples. By S. R. Taylor. Pp. $x i x+372$. (Pergamon: New York and Oxford, 1975.) $\$ 16.50 ; £ 6.90$.

THE technological tour-de-force in landing men on the Moon in the six missions Apollo 11, 12, 14, 15, 16 and 17 and the drama of Apollo 13-in particular the extreme sophistication of the systems control-has perhaps overshadowed the scientific achievements in the study of the returned lava, breccia and soil samples and the observations from experimental apparatus, some still functioning on the Moon. How appropriate it would be in this bicentennial year of the US if NASA produced a history of this unprecedented scientific project: by what alchemy did imaginative planning emerge from committees of scientists and admini strators in Washington. But in some ways the very triumph of the scientific collaboration has been buried in vast conference proceedings; and it has been left to Dr Ross Taylor to give a most admirable answer to the question insistently asked "What really did this proiect discover about the Moon?".

Many of the conclusions reached or reinforced by evidence brought back by the Apollo proiect are set out clearly by $\mathrm{Dr}$ Taylor, and the book is a remark- able one: no scientist could fail to be fascinated and even those most learned about the Moon will learn something more. One miracle, however, was not achieved by the planners of the Apollo project : in spite of all attempts it has not entirely succeeded in bringing about an easy communication between petrologists and geochemists on the one hand and geophysicists on the other. In the many lunar conferences once the former begins talking about ferropseudobrookite and hedenbergite-ferrohedenbergite, the eyes of the latter become glazed; and when the latter begin speaking of electromagnetic transfer functions and magnetic Reynolds numbers, the conference room empties noticeably. In his treatment of the physics of the Moon, mainly in chapter 6 (only 29 pages), Dr Taylor falls from the high standard elsewhere.

Yet it was in this field that three of the most surprising and unexpected discoveries were made-positive anomalies in gravitational field occurring over the circular maria, remanent magnetisation of the returned specimens, along with magnetic anomalies in the lunar crust and moonquakes occurring predominantly at apogee and perigee. Dr Taylor does not treat these discoveries-fundamental to any understanding of the Moon's interior and its evolution-in the depth they deserve. For example in lunar magnetism he relies on a review, which he says provides "appropriate skeptical comments on current theories." Although some ideas which have been suggested to explain this unexpected finding are plausible-Velikovsky's prediction that the lunar surface was magnetised in 3,000 BC-some theories merit serious consideration. But some inconclusiveness is only to be expected when a new phenomenon is discovered. Observations that can be immediately understood in terms of currently accepted orthodoxy are not of so much interest as those which open up new approaches.

The fundamental significance of the non-hydrostatic figure of the Moon is ignored and thermal convection occurring by solid-state creep in its interior is not mentioned. The essential requirement of a theory of masconsthat is, that extra mass, whether lava or not, must be brought by lateral transport into the circular mare areais not made clear. The implications of the remarkable correlation between the occurrence of moonquakes and the tides, and the analogous effect with regard to the enigmatic lunar transient events, are also not brought out.

S. K. Runcorn 